\title{
Thermoset modified with polyethersulfone: characterization and control of the morphology.
}

\author{
Eléonore Mathis ${ }^{1}$, Marie-Laure Michon ${ }^{2}$, Claude Billaud ${ }^{3}$, Pauline Grau $^{2}$, Anthony Bocahut ${ }^{2}$, Caroll \\ Vergelati ${ }^{1}$, Didier R. Long $^{1}$
}

${ }^{1}$ LPMA, Laboratoire des Polymères et Matériaux Avancés, UMR 5268 Solvay/CNRS, Solvay in Axel'One, 87 avenue des Frères Perret 69192, Saint-Fons, France

${ }^{2}$ Solvay Research and Innovation, 85 avenue des Frères Perret 69192, Saint-Fons, France

${ }^{3}$ Solvay Composite Materials, R420 The Wilton Centre, Redcar TS10 4RF, UK

*Present address: Solvay Specialty Polymers, Viale Lombardia, 20, 20021 Bollate, Italia

Correspondence to: Eléonore Mathis (E-mail: eleonoremathis29@gmail.com), Didier R. Long (E-mail: didier.r.long@gmail.com)

\begin{abstract}
Thermoset (TS) epoxy resins can be toughened with a thermoplastic (TP) for high-performance applications. The final structure morphology has to be controlled in order to achieve high mechanical properties and high impact resistance. Four polyethersulfone-modified epoxy resins are considered. They consist of different epoxy monomer structure (TGAP, triglycidyl-p-aminophenol and TGDDM, tetraglycidyl diaminodiphenylmethane) and a fixed amount of thermoplastic, they are cured with two different amounts of curing agent. A reaction-induced phase separation occurs for all formulations generating morphologies, different in shapes and scales. The aim is to control the final morphology and in particular its dominant length scale. This morphology depends on the phase separation process, from the initiation to its final stage. The initiation relies on the relative miscibility of the components and on the stoichiometry between epoxy and curing agent. The kinetics depends on the viscosity of the systems. The different morphologies are characterized by electron microscopy or neutron scattering. Dynamic mechanical analysis allows confirming the presence of a phase separation even when it is not observable by electron microscopy. Vermicular morphologies with few hundreds nanometer width are obtained for the systems containing the TGAP as epoxy monomer. Systems formulated with TGDDM presents morphologies on much smaller scale of order a few tens of nanometers. We interpret the different sizes of the morphologies as a consequence of a larger viscosity for the TGDDM systems as compared to the TGAP ones rather than by a latter initiation of phase separation.
\end{abstract}

\section{INTRODUCTION}

High-performance epoxy matrices have been used for decades in fiber-reinforced composites for aerospace industry. It is adopted today also by the automotive industry. Because of their light-weight, they are used to replace metals. Thermoset resins show high stiffness and strength as well as good thermal and chemical stability. However, their high cross-link density make them very brittle and of poor impact and crack growth resistances. Their toughening by the addition of thermoplastic depends directly on the final morphology. By monitoring the micro-structure of the cured system, the toughness can be optimized for composite applications [1, 2]. For polymer blends, it was shown in the literature that a significant improvement of the impact resistance is obtained when tougheners have phase 
separated to form a morphology at the submicron scale $[3,4]$. Specifically in polyethersulfone/epoxy blends, better fracture toughness was observed for systems presenting bicontinuous morphology $[5,6]$.

Since the work of Bucknall and Partridge in 1983 [7], polyethersulfones are used as toughener in epoxy systems $[8,9]$. Toughening was obtained with samples exhibiting a twophase morphology. The TP content was found to be one of the most important factor influencing final morphologies and related mechanical properties [1]. Upon increasing the TP content, the final resin structure can evolve from sea-island morphology (TP-rich nodules dispersed in TS-rich matrix) to phase inverted morphology (TS-rich domains dispersed in a TPrich matrix) [10]. Increasing the thermoplastic molecular weight leads to a phase separation at lower epoxy conversion [11, 12]. Rosetti et al. [13] showed that a hydroxyl reactive end-group on the thermoplastic leads to finer morphologies as it may react with epoxy group, compared to non-reactive thermoplastic of the same molecular weight [14]. The phase separation occurs also later during the curing process. In another study, it was shown that the presence of reactive end-group on TP favoured a stronger interface between TP and epoxy and improve the toughness of the material [15]. The relative miscibility of the components in the initial systems is also described as a parameter of choice that influences the phase separation behaviour $[8,14]$. The miscibility of the components is characterized by the FloryHuggins $\chi$ parameter [16].

Enhancing toughness properties such as impact resistance by the control of the final resin morphology still remains a scientific challenge. We propose a methodology to control the final morphology by combining various approaches with regard to the affinity of the constituents characterized by their $\chi$ parameters, the viscosity of the systems, the kinetics of epoxy curing and the gel point determination. We consider four different polyethersulfone/epoxy based formulations. Systems are prepared with two different epoxy monomers: the TGAP, triglycidyl-p-aminophenol or the TGDDM, tetraglycidyl diaminodiphenylmethane, and with two different amounts of curing agent. The influence of the initial resin composition on the onset temperature of phase separation, as well as on the phase separation kinetics is discussed. A soluble thermoplastic technology is used for preparing the resin. The chosen polyethersulfone is soluble in the thermoset precursors and phase separates upon curing [17]. Phase separation evolves until the gelation state of the system which stops the evolution of the morphology $[18,19]$. After the gel point, the compositions of the thermoplastic-rich phase and of the epoxy-rich phase may still evolve. To study these effects, the curing reaction is characterized in situ through thermal and rheological analyses. Cure kinetics, gel point temperatures and phase separation mechanisms are studied. Morphology of the cured systems are analyzed by electron microscopy and further characterized by dynamic mechanical thermal analysis and smallangle neutron scattering if on the nano-scale $(<$ $100 \mathrm{~nm})$.

TGAP-systems result on vermicular morphology with few hundreds nanometer width. TGDDMsystems result on small nodular morphologies as the high viscosity of the resin system slows down the growth of nodules. We discuss the effect of the components affinity and of the viscosity of the four different formulations on the final morphology of our samples.

\section{EXPERIMENTAL}

\section{Materials}

The epoxy resin precursors were kindly supplied by Huntsman Advanced Materials, Basel (Switzerland): triglycidyl-p-aminophenol (TGAP, Araldite MY0510), tetraglycidyl diaminodiphenylmethane (TGDDM, Araldite MY721) and 4, 4'-diaminodiphenylsulfone (4, 4'DDS, Aradur 9664-1). TGDDM has an epoxy 
equivalent weight of $111-117 \mathrm{~g} \mathrm{eq}^{-1}$ and a viscosity of $3000-6000 \mathrm{cP}$ (at $25^{\circ} \mathrm{C}$ ). TGAP has an epoxy equivalent weight of $95-107 \mathrm{~g} \mathrm{eq}^{-1}$ and a viscosity of $550-850 \mathrm{cP}$ (at $25^{\circ} \mathrm{C}$ ). The modifier is a hydroxyl-functionalized polyethersulfone (PES, PES-5003P from Sumitomo) with a number average molecular weight of $24,000 \mathrm{~g} \mathrm{~mol}^{-1}$ and a $\mathrm{Tg}$ of $232^{\circ} \mathrm{C}$.
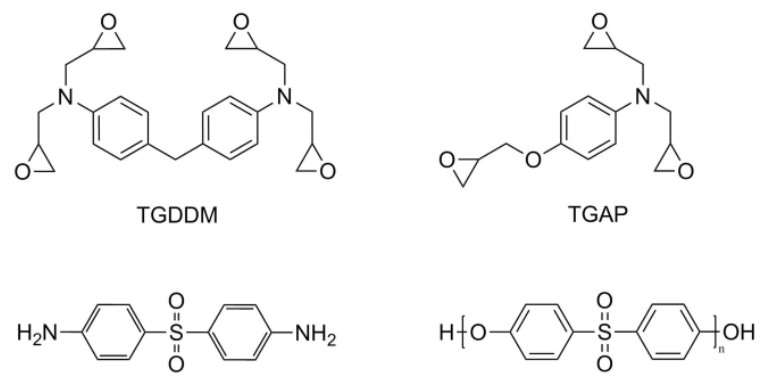

4, 4'-DDS

PES

FIGURE 1 Structure of the resin components.

\section{Determination of interaction parameters}

As introduced by Pascault in 1995[8], the initial miscibility of epoxy monomers with modifier influences the final morphology. In order to compare the miscibility behavior of blends in their initial state, Flory interaction parameters [20] between two components are calculated. Polymer solubility parameters are estimated by using the group contribution theory. The Hoy method [21] is used to tabulate the group contributions. This method assumes a similarity between the solubility parameter of a polymer and the solubility parameter of its repeat unit. The solubility parameter $\delta$ of a component is determined by summing the different contributions of its chemical groups, i.e. the dispersive contribution, the polar contribution and the hydrogen bonding contribution. The corresponding values for each group are provided in the Tables of the Hoy method [21]. The interaction parameters between two components are calculated by using Equation 1. $\chi_{1-2}$ is the Flory interaction parameter, $\delta_{1}$ and $\delta_{2}$ are the Hoy solubility parameters in for each component in $\left(\mathrm{J} \mathrm{cm}^{-3}\right)^{1 / 2}, \mathrm{~V}_{1}$ and $\mathrm{V}_{2}$ are their respective volume in $\mathrm{cm}^{3} \mathrm{~mol}^{-1}, \mathrm{R}$ is the gas constant in $\mathrm{J} \mathrm{mol}^{-1} \mathrm{~K}^{-1}$ and $\mathrm{T}$ is the temperature in $\mathrm{K}$.

$$
\chi_{1-2}=\frac{\left(\delta_{1}-\delta_{2}\right)^{2}\left(\mathrm{~V}_{1} \mathrm{~V}_{2}\right)^{1 / 2}}{\mathrm{RT}}(\text { Equation } 1)
$$

Results for a temperature of $120^{\circ} \mathrm{C}$ are given in Table 1 . No reaction is monitored at that stage yet. The calculation of $\chi$ assumes that the volume of the system is constant upon mixing.

TABLE 1 Flory interaction parameters in initial uncured systems.

\begin{tabular}{llll}
\hline System & $\begin{array}{l}\text { Epoxy } \\
\text { monomers }\end{array}$ & Thermoplastic & $\begin{array}{l}\text { Flory interaction } \\
\text { parameter } \chi \text { at } \\
120{ }^{\circ} \mathrm{C}\end{array}$ \\
\hline A & TGDDM & PES-5003P & 0.27 \\
B & TGAP & 0.03 \\
\hline
\end{tabular}

Note that interaction parameters are calculated for binary systems. A supplementary step could allow to obtain the phase diagram of ternary systems as developed by Kurata [16]. For our study, it allows to obtain qualitative comparisons between the systems $A$ and $B$ composed of epoxy monomers with different structures. It shows that TGAP is highly miscible with the TP (low $\chi$ value); on the opposite TGDDM is not a good solvent for the TP (high $\chi$ value).

From the determination of the interaction parameters of the components in the initial state, it is possible to determine the onset of phase separation between the epoxy oligomers in formation and the polyethersulfone. It is based on the principle that phase separation occurs when $\mathrm{N} \chi>0.5$, where $\mathrm{N}$ is the number of reacted monomers and $\chi$ the initial Flory interaction parameter. Upon curing through a stepwise polymerization, the oligomer molecular weight grows: the number of the repeat units per oligomer increases until reaching the gel point when the value of $\mathrm{N}$ tends to infinity. Gelation is a critical phenomenon described by percolation theory $[22,23]$. Near gel-point, the size $\mathrm{N}$ of epoxy aggregates can be calculated by the following (Equation 2): 


$$
\mathrm{N} \sim\left|\frac{\mathrm{p}-\mathrm{p}_{\mathrm{c}}}{\mathrm{p}_{\mathrm{c}}}\right|^{-1 / \sigma} \text { (Equation 2) }
$$

where $\sigma$ is a percolation exponent with the value of 0.45 for a system in dimension $3, p_{c}$ is the epoxy conversion at gel point and $\mathrm{p}$ is the epoxy conversion. Epoxy conversion at gel point is determined by using dynamic rheology as it will be explained in section 3.2. After calculation of $\mathrm{N}$ during epoxy curing, the epoxy conversion at phase separation is approximated. The results are shown in Table 4.

\section{Sample preparation}

Four systems are formulated. Systems $A$ are prepared by mixing TGDDM with 4, 4'-DDS: A1 with a reactive amino hydrogen to epoxy stoichiometric ratio (NH/Ep) of 0.9 and $\mathrm{A} 2$ with $\mathrm{NH} / \mathrm{Ep}$ of 1.15 . Systems $B$ are synthetized by mixing TGAP with 4, 4'-DDS: B1 with NH/Ep of 0.9 and B2 with NH/Ep of 1.15. The thermoplastic content is fixed to 15 weight percent (wt \%) for all systems.

\begin{tabular}{lllll}
\multicolumn{5}{c}{ TABLE 2 Formulations investigated in this study } \\
\hline System & $\begin{array}{l}\text { Epoxy } \\
\text { monomers }\end{array}$ & $\begin{array}{l}\text { Curing } \\
\text { agent }\end{array}$ & $\begin{array}{l}\text { Stoichiometry } \\
\text { NH/Ep }\end{array}$ & $\begin{array}{l}\text { Thermoplastic } \\
\text { (15wt \%) }\end{array}$ \\
\hline A1 & TGDDM & 0.9 & \\
A2 & & $\begin{array}{l}4,4^{\prime}- \\
\text { DDS }\end{array}$ & 1.15 & PES-5003P \\
B1 & TGAP & & 0.9 & \\
B2 & & & 1.15 & \\
\hline
\end{tabular}

Systems are prepared by dissolving at first the thermoplastic in the epoxy monomer of choice at $140{ }^{\circ} \mathrm{C}$; 4 hours are needed for the TGDDM systems while only 2 hours are sufficient for the TGAP systems. The 4, 4'-DDS curing agent is then added to the homogenized blend of epoxy and TP at $120{ }^{\circ} \mathrm{C}$. The mixture is further stirred to achieve a partial dissolution of the $4,4^{\prime}$-DDS. Before curing, the samples are degassed under vacuum at $90{ }^{\circ} \mathrm{C}$ for 2 hours. Finally for all systems, similar cure profiles are used with first a temperature ramp of $1{ }^{\circ} \mathrm{C} / \mathrm{min}$ up to $180{ }^{\circ} \mathrm{C}$ and a plateau maintained at $180{ }^{\circ} \mathrm{C}$ to achieve the resin full cure. For all systems, it is checked that no reaction occurs during degassing and that the DDS is dissolved, by running DSC analyses on non-degassed and degassed samples. The final degree of cure is determined by running DSC analyses on the cured sample and by comparing the residual heat flow to the total heat flow determined from the degassed samples. It evolves from $90 \%$ for TGDDMsystems to $95 \%$ for TGAP-systems.

\section{Material characterization}

\section{Physical and chemical characterization of system}

\section{In-situ kinetic study of epoxy curing by DSC}

Thermal analyses are performed with a Q2000 Differential Scanning Calorimeter (TA Instruments). A sample of uncured resin (6 to 8 $\mathrm{mg}$ ) is placed in an aluminum pan hermetically sealed and is analyzed from -50 to $310^{\circ} \mathrm{C}$ at $1{ }^{\circ} \mathrm{C}$

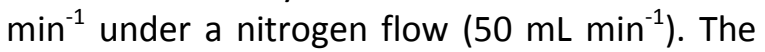
uncured resin glass transition temperature and the heat flow of the exothermic curing process are measured. Degree of epoxy conversion upon curing is calculated from the enthalpy of reaction of the exotherm peak (Equation 3 ). $\mathrm{H}_{\mathrm{T}}$ is the integrated area of the exotherm at temperature $\mathrm{T}, \mathrm{H}_{\text {Total }}$ is the total area of the exotherm, i.e. the total enthalpy of reaction, and $T_{0}$ is the temperature at the onset of the exotherm.

$$
\left.\alpha_{\mathrm{T}}=\frac{\int_{\mathrm{T}_{0}}^{\mathrm{T}} \frac{\mathrm{dH}_{\mathrm{T}}}{\mathrm{dT}} \mathrm{dT}}{\Delta \mathrm{H}_{\mathrm{Total}}} \text { (Equation } 3\right)
$$

Kinetic studies are made in triplicate in order to determine accurately the degree of conversion upon curing.

\section{Determination of gel point by dynamic rheology}

Dynamic rheological measurements are performed by using an oscillation rheometer (ARES-G2 rheometer, TA Instruments) equipped with disposable parallel plate geometry of 25 $\mathrm{mm}$ in diameter. Gap is adjusted using a constant force, and auto-strain adjustment from 0.01 to $50 \%$ is used which corresponds to the linear viscoelastic regime. Multiwave 
rheological measurements are used on a nonisothermal test from 80 to $200{ }^{\circ} \mathrm{C}$ at $1{ }^{\circ} \mathrm{C} \mathrm{min}$. Storage modulus $\left(G^{\prime}\right)$, loss modulus $\left(G^{\prime \prime}\right)$ and complex viscosity $\left(\eta^{*}\right)$ of melts are collected. Gel point is determined as the crossover of tan $\delta$ curves for several frequencies (data extracted from the multiwave experiment).

\section{Phase separation characterization on cured system}

\section{Morphology observation by SEM}

The morphology of cured samples is analyzed by Scanning Electron Microscopy by using a Zeiss Ultra 55 microscope. The TP-rich phase is revealed by the acid phosphotungstic staining of $300 \mathrm{~nm}$ thick cross-sections of cured specimens prepared by microtomy at room temperature. An acid etching is also used as a second method in order to confirm the morphology obtained by the staining method. Polished samples are soaked in a sulfuric acid solution which removed the TP-rich phase. All samples are metallized with Platinum prior being observed.

\section{Morphology observation by TEM}

The morphology of cured samples is analyzed by Transmission Electron Microscopy by using a MET JEOL 1400. Ultrathin cross-sections from 50 to $100 \mathrm{~nm}$ in thickness are prepared by microtomy at room temperature. The resin micro-structure is revealed by the same staining method.

\section{Determination of phase separation by DMTA}

Dynamic mechanical thermal analysis (DMTA) measurements are performed on cured resins by using a rheometer Scientific analyzer RSAG2 (Ta instrument). A part of resin disc has been cut in the dimensions $40 \times 10 \times 3 \mathrm{~mm}^{3}$. The mechanical relaxations were characterized by using the three-point bending method. After determining the linear regime, a strain limit is fixed ( $5 \mathrm{E}-3 \%$ ) and curves are recorded at $10 \mathrm{~Hz}$ during heating from $140{ }^{\circ} \mathrm{C}$ to $300{ }^{\circ} \mathrm{C}$ at $2{ }^{\circ} \mathrm{C}$ $\min ^{-1}$. Relaxation temperatures are determined from the peak of $\tan \delta$ plot.

Characterization of morphology by small-angle neutron scattering

Small-angle neutron scattering (SANS) are carried out at beamline D11 at Institut LaueLangevin (ILL, Grenoble, France). Three configurations are used: $5.5 \mathrm{~m} / 5.3 \AA$, $28 \mathrm{~m} / 5.3$ $\AA$, $37 \mathrm{~m} / 12 \AA$, covering a size range from 5 to $1.2 \mathrm{E}+3 \mathrm{~nm}$. The sample cell is made of an aluminum mold of $20 \mathrm{~mm}$ in diameter where samples are poured after degassing. Resins are cured in an oven and quenched at different time of the curing cycle. Samples are then stored in freezer before being studied during the beam shift on the reactor. The observed scattering intensities are corrected for incident neutron spectrum, sample transmission and sample thickness, background scattering. The incoherent scattering is subtracted to the intensity as the invariant intensity at high q.

\section{RESULTS AND DISCUSSION}

\section{In-situ kinetic study of epoxy curing}

For all resin systems, the profiles of epoxy conversion are determined by DSC nonisothermal analyses at $1{ }^{\circ} \mathrm{C} \mathrm{min}^{-1}$ to mimic the chosen curing cycle. Samples are heated up to $310{ }^{\circ} \mathrm{C}$ in order to achieve a complete epoxy conversion $(\alpha=1)$ and to determine the total area of the exotherm $\mathrm{H}_{\text {Total }}$. It is then possible to plot the degree of cure along temperature (or time) by using Equation 3 (Figure 2). For each system, the gel point is determined by dynamic rheology as explained in section 3.2. The gel point is indicated by crosses in Figure 2 . 


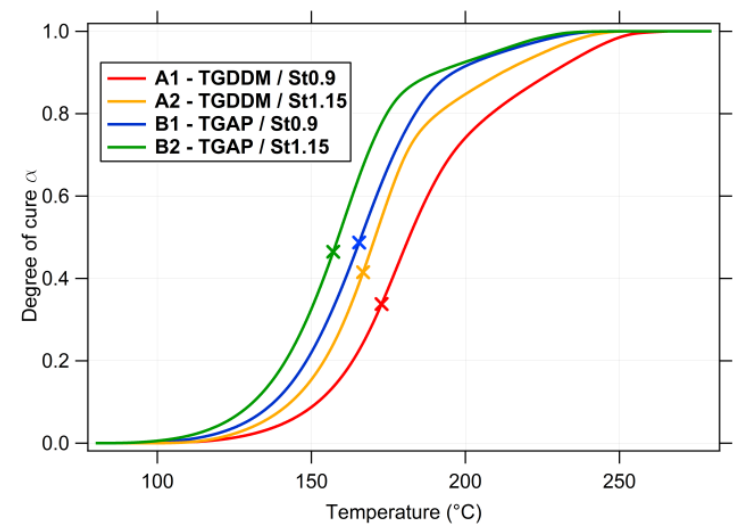

FIGURE 2 Evolution of the degree of cure along temperature ramp at $1{ }^{\circ} \mathrm{C} \mathrm{min}{ }^{-1}$. The point marked by a cross represents the gel point.

Differences between the cure kinetics of the 4 formulations are observable. Systems containing TGDDM ( $\mathrm{A} 1$ and $\mathrm{A} 2$ ) have a slower kinetic than systems with TGAP throughout the curing process and particularly close to their gel points. TGDDM systems have a higher viscosity than TGAP systems (Figure 4) which may reduce the monomers and oligomers mobility and thus the kinetics of reaction. Moreover, TGDDM epoxy monomer has a functionality of 4 which may induce more steric hindrance on its reactive epoxy groups than TGAP which has a functionality of 3, particularly after some epoxy branched. Also because of its highest functionality, vitrification should appear earlier in systems $A 1$ and $A 2$ which then should reduce chain mobility. After vitrification, reaction kinetics is controlled by diffusion. As an indication of this change of kinetic control, the changes of slope on curves $A 1$ and $A 2$ are observable around $70 \%$ of curing degree. Swanson et al. [24] explained the same influence of the monomer functionality and the steric hindrance by comparing the kinetic of epoxy conversion by near-infrared spectroscopy of three systems with TGAP, TGDDM or difunctional epoxy (DGEBA). Another tendency in our results is that the stoichiometry of the system influences the cure kinetics whatever the monomer epoxy is. As expected, epoxy conversion is faster all along the curing process for systems with excess of curing agent (A2, B2) because more reactive amine groups are present and accessible for the reaction with epoxy groups.

\section{Determination of gel point}

Gel point is often determined as the crossover of the elastic $\left(G^{\prime}\right)$ and loss $\left(G^{\prime \prime}\right)$ moduli for isothermal experiments [25] as well as nonisothermal experiments [26] for a fixed frequency test. It is a convenient approach for some network polymers for which $\tan \delta=G^{\prime \prime} / G^{\prime}$ $=1$ but it is not a general definition of the gel point as explained by Winter [27], who recommends to consider the $\tan \delta$ curves crossover (obtained from different frequency tests) for determining the gel point. Indeed this critical transition does not depend on the frequency of the test contrary to the vitrification phenomena which is frequency test dependent [28]. Gel point according to Winter criterion was determined by Cicala [29] by plotting $\tan \delta$ as a function of the time using a multiwave test. We apply the same method but in dynamic regime, with a temperature ramp at a rate of $1{ }^{\circ} \mathrm{C} \mathrm{min}^{-1}$, using a parallel plate geometry. The multiwave rheological measurement allows to apply the sum of a fundamental frequency and different harmonics each having individual strain amplitudes in one single test. The sum of the strain amplitudes require to stay into the linear viscoelastic regime. Then the individual responses at each discrete frequency can be obtained through a discrete Fourier transform. Results of the multiwave tests with $1,3,6,10 \mathrm{~Hz}$ as discrete frequency for systems B1 are plotted in Figure 3. 

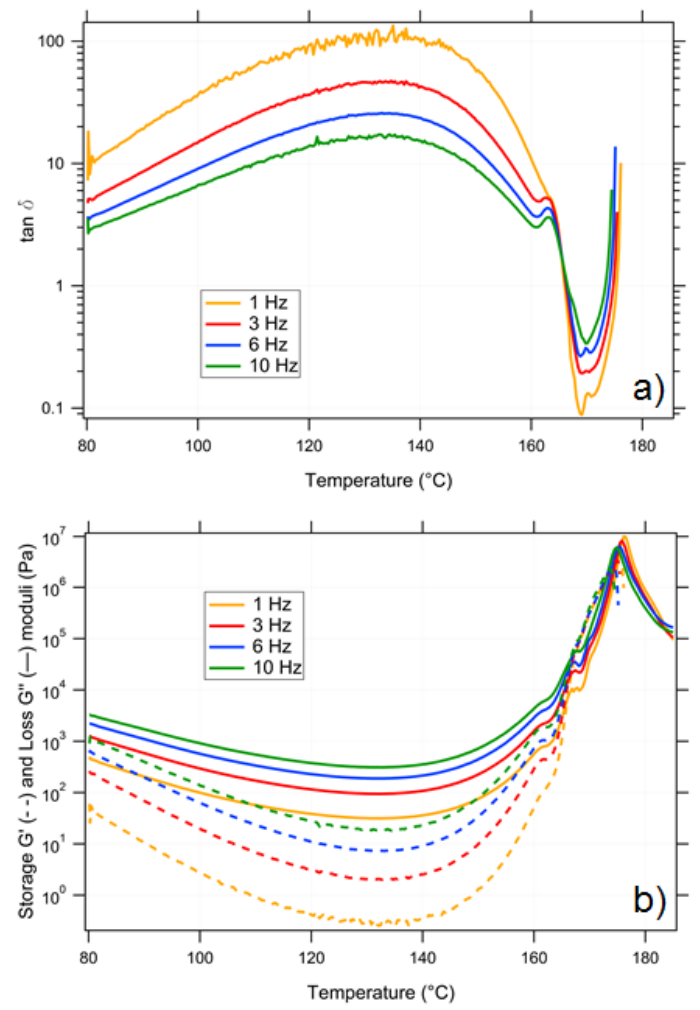

FIGURE 3 Storage and loss moduli (a) and $\tan \delta$ curves (b) for 4 frequencies for system B1.

The temperature at the gel point is determined as the $\tan \delta$ crossover and the corresponding degree of cure is calculated from previous DSC experiments. Degree of cure at gel point is also calculated from the Flory-Stockmayer equation [20] (Equation 4), where $f_{e}$ and $f_{a}$ are the epoxy and amine functionalities respectively and $s$ the stoichiometry. This calculation only considers the functionality but not the possible difference in their respective reactivity and it excludes the possibility of intramolecular reaction.

$$
\alpha_{\text {gel }}=\left(\frac{\mathrm{s}}{\left(\mathrm{f}_{\mathrm{e}}-1\right)\left(\mathrm{f}_{\mathrm{a}}-1\right)}\right)^{1 / 2} \text { (Equation 4) }
$$

All dynamic rheology results are indicated in Table 3, as well as the theoretical degree of cure at gel point for comparisons.

TABLE 3 Gel point characterization for all systems

\begin{tabular}{lllll}
\hline \multirow{3}{*}{ System } & $\begin{array}{l}\text { Gel point } \\
\text { temperature } \\
\left({ }^{\circ} \mathrm{C}\right)\end{array}$ & $\begin{array}{l}\text { Tan } \delta \\
\text { at gel } \\
\text { point }\end{array}$ & $\begin{array}{l}\text { Degree } \\
\text { of cure at } \\
\text { gel point }\end{array}$ & $\begin{array}{l}\text { Theoretical } \\
\text { degree of } \\
\text { cure at gel } \\
\text { point }\end{array}$ \\
\hline
\end{tabular}

\begin{tabular}{lllll}
\hline & & & & (Flory) \\
\hline A1 & 172.8 & 0.90 & 0.33 & 0.32 \\
A2 & 166.8 & 0.98 & 0.42 & 0.36 \\
B1 & 165.5 & 1.78 & 0.49 & 0.39 \\
B2 & 157.1 & 1.76 & 0.47 & 0.44 \\
\hline
\end{tabular}

Since steric hindrance effects may affect the reactivity of the different functional group in our system, and intramolecular reactions may occur, the experimental results assume larger values than those predicted by the FloryStockmayer theory, except for A1 and B2 which seem to agree more with these predictions. Experimental and theoretical results show that gel points occur at lower epoxy conversion for TGDDM-systems (A1 and A2) which can be explained by its higher functionality: the growing molecules build a percolating network faster. It is noticeable that the value of $\tan \delta$ at gelation is not always equal to 1 . As defined by Winter and Chambon [27], it depends on the resin composition. The tan $\delta$ value provides information on the network rigidity: $\tan \delta$ increases with the network stiffness. Thus, systems containing TGAP (B1, B2) epoxy monomers show a structure at the gel point with a higher stiffness than TGDDM systems (A1, A2), which is consistent with a higher epoxy conversion at gel point for those systems. The determination of the gel point upon curing has to be discussed in relation with the phase separation onset in order to access the extent of phase separation and to understand the finally obtained morphology.

Onset of phase separation can be determined by dynamic rheology. It has been often studied by running isothermal experiments. Cicala et al. $[29,30]$ compared viscosity fluctuations in different epoxy/coPES blends cured at different temperatures. For 15 wt \% of thermoplastic, they recorded a sudden decrease in viscosity attributed to the demixing of a highly viscous TP. The same change of slope of the viscosity profiles is clearly observed in our study for the 
systems B1 and B2 at a constant temperature for all frequencies. The viscosity profile results from two contributions: the formation of a TPrich phase (decrease in TP content present in the epoxy phase formed coincidentally) and the formation of the epoxy network (increase in molecular weight). For a better visualization, we plot the storage modulus curves in Figure 4, for the $10 \mathrm{~Hz}$ experiments. The gel point determined by the tan $\delta$ crossover is indicated by a cross and the approximate onset of phase separation is indicated by an arrow with the corresponding epoxy conversion. However no clear changes in viscosity are observable in systems $A 1$ and $A 2$. It may indicate that no phase separation occurs or that the contribution of phase separation on the viscosity is less important than the contribution of the network development. Note that the signature of a phase separation in dynamic rheology is accessible if the experiment occurs in a certain window of study which depends on the test frequency, the size of the interface, the viscosity ratio between the two phases. It is explained below that two phases are detected in all cured systems by DMTA, so that phase separation is confirmed for all systems but not discerned on systems A by rheology only.

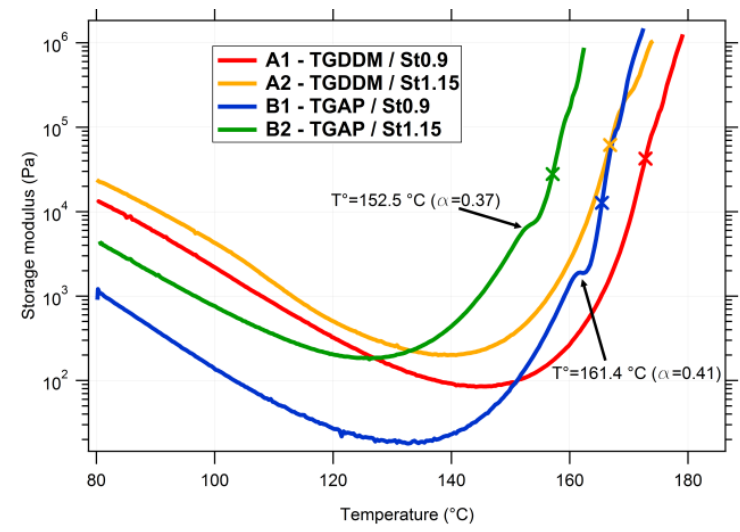

FIGURE 4 Evolution of storage modulus along curing for all systems

In order to further explain the resulting morphology of the different systems, a theoretical estimate of epoxy conversion at phase separation based on scaling model of gelation is used (Equation 2). It allows for the calculation of the number of monomers branched at phase separation (when $\mathrm{N} \chi>0.5$ ) and therefore to determine the corresponding epoxy conversion value. The results are shown below in Table 4.

TABLE 4 Estimation of epoxy conversion at phase separation based on scaling model of gelation.

\begin{tabular}{ll}
\hline System & $\begin{array}{l}\text { Epoxy conversion at } \\
\text { phase separation }\end{array}$ \\
\hline A1 & 0.08 \\
A2 & 0.11 \\
B1 & 0.40 \\
B2 & 0.34 \\
\hline
\end{tabular}

It is important to note that the end-group of the PES5003P can react with epoxy as studied by Cheng et al. [31] and mentioned by Rosetti [13]. Thus an epoxy-PES block copolymer is formed which is more soluble with epoxy than the PES. This enhanced compatibility delays the phase separation. So epoxy conversions at phase separation may be higher than those calculated above. Nevertheless we observe that the theoretical estimates regarding the epoxy conversion at phase separation for B1 and B2 are close to but slightly lower than the experimental values $(0.41$ and 0.37 respectively). Thus we assume that the epoxy conversion at phase separation calculated for $A 1$ and $A 2$ are also conservative estimates.

\section{Resulting morphology of the epoxy/polyethersulfone blends}

After curing, the morphology of all formulations is characterized. The reaction-induced phase separation in TP/TS blends is due to the increase of the molecular weight of the growing thermoset. The morphologies observed which have a definite length scale and are homogeneous on larger scales are relevant to spinodal decomposition rather than nucleation and growth mechanisms. Spinodal decomposition occurs when the system goes into the unstable region of its phase diagram $[12,32]$. The resulting morphology may be cocontinuous or sea-island if a percolation-to- 
cluster mechanism permits to reduce the interfacial energy of the system $[9,11]$. Scanning Electron Microscopy (SEM) on cured systems after chemically labelling the TP-rich phase permits to bring out clearly the phase separation in systems B. TP-rich phase is dispersed in the epoxy matrix. In Figure 5 the morphologies obtained for systems B are presented.

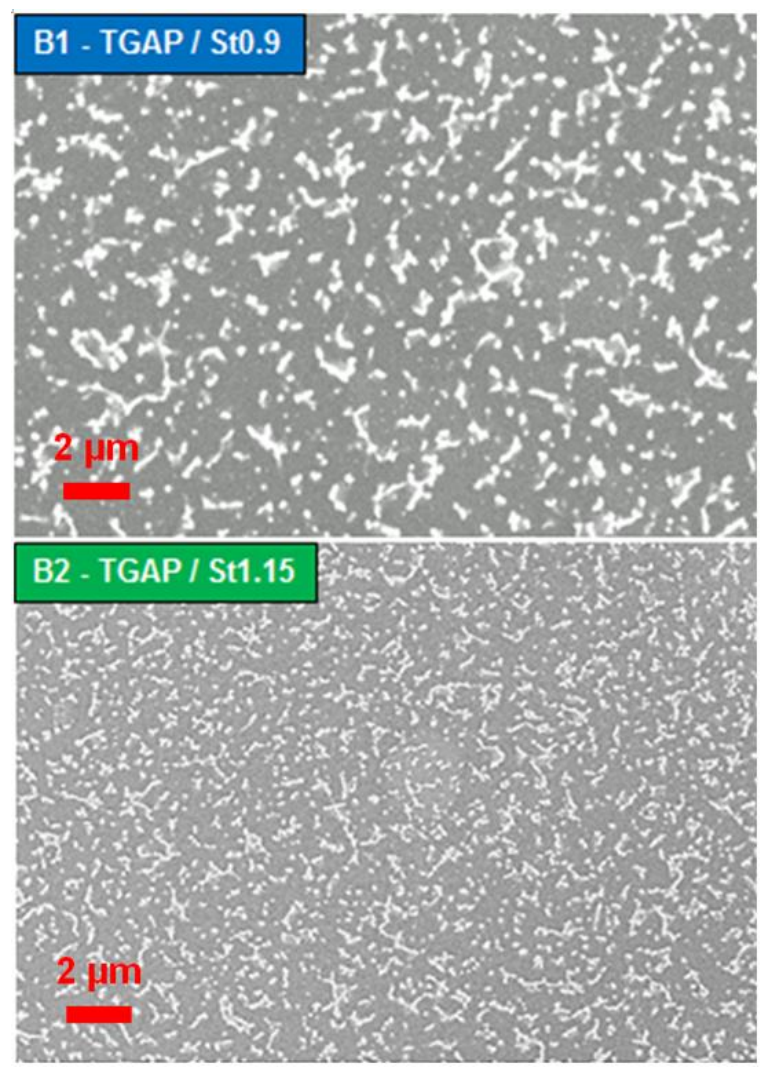

FIGURE 5 SEM observations of systems $B$. The TP-rich phase is chemically stained and appears as bright domain. Scale bar $2 \mu \mathrm{m}$.

Systems B have also been prepared for SEM observation by acid etching method in which the thermoplastic is dissolved, in order to confirm the observed morphologies.
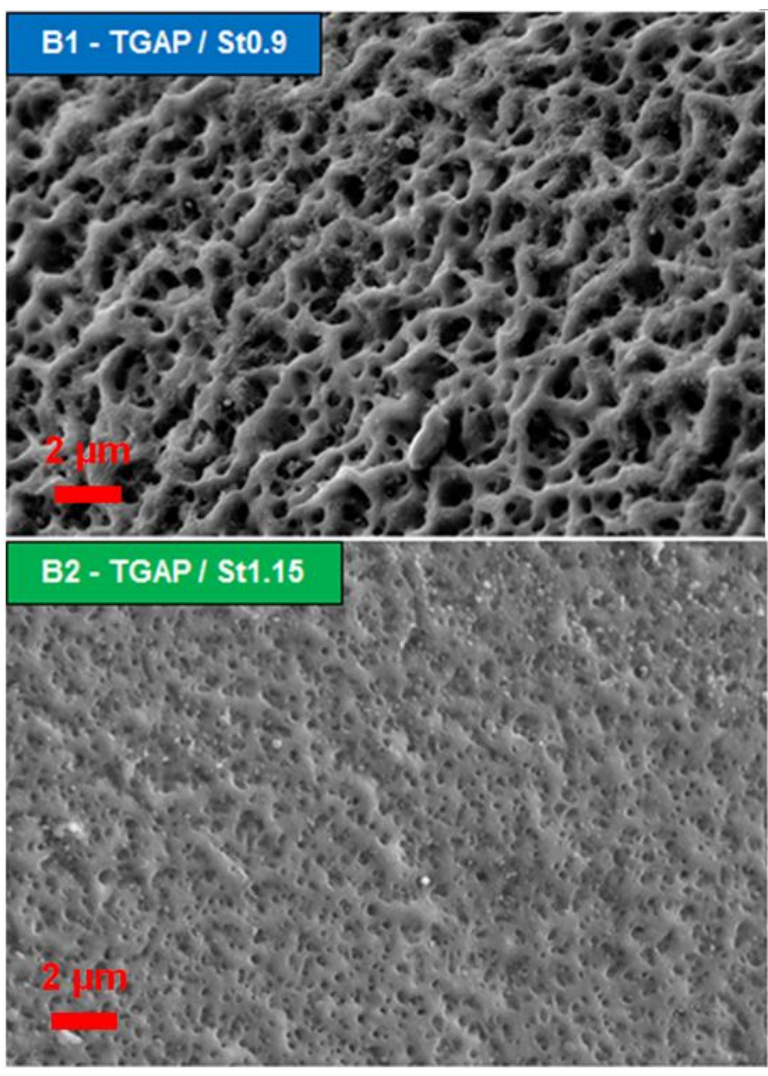

FIGURE 6 SEM observation of the systems B.

The TP-rich phase is dissolved by acid etching method. Scale bar $2 \mu \mathrm{m}$.

Systems B show vermicular morphologies which can also be described as discontinuous morphologies. We propose that this type of morphology is due to a swelling of TP-noduli by the TGAP which is highly compatible with the thermoplastic so that the amount of TP-noduli is close to the percolation threshold [23]. Size wise, it is interesting to compare the width of the vermicular morphology which is of submicron scale, while the lengths can cover a few micro-meters when nodules coalesce. The average width of the PES-rich domains is about $250 \mathrm{~nm}$ for the formulation cured at a stoichiometry of 0.9 (B1), while $150 \mathrm{~nm}$ width is observed for the formulation with amine excess (B2). Furthermore the nodules of the B2 resin appear to have less coalesced. Rosetti and al. [13] obtained vermicular morphologies for epoxy systems (blends of di and tri-functional epoxies) modified with 13 wt $\%$ of a similar polyethersulfone but with non-reactive end- 
groups. The scales are slightly smaller in our systems which can be explained by the presence of the $-\mathrm{OH}$ end-group on the polyethersulfone which may react with epoxy [1] and so increase its compatibility and delayed phase separation.

Also, it is noteworthy that the staining method permits to obtain information on the inside of TP-rich phase at high magnification. In system B2, nodules of epoxy are observable with a diameter of about $50-60 \mathrm{~nm}$. We interpret this effect as a consequence of a secondary phase separation within TP-rich domains when curing goes on. It is likely that this second phase separation process takes place by nucleation and growth.

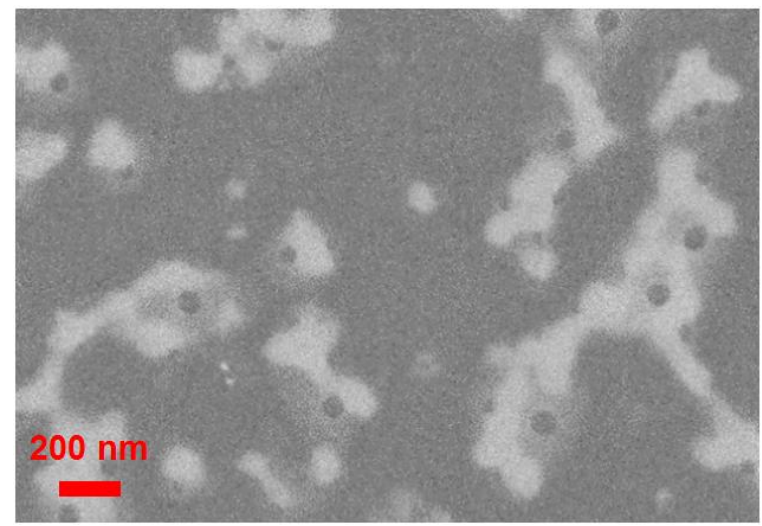

FIGURE 7 SEM observations of system B2 at high magnification. Scale bar $200 \mathrm{~nm}$.

To improve the characterization of the morphology, Transmission Electron Microscopy (TEM) is performed on cured systems $A$ and $B$ after chemically staining the TP-rich phase. The observations are done under magnificence from $10 \mathrm{k}$ to $300 \mathrm{k}$. Detailed images of the morphologies of systems B1 and B2 are obtained (Figure 8).

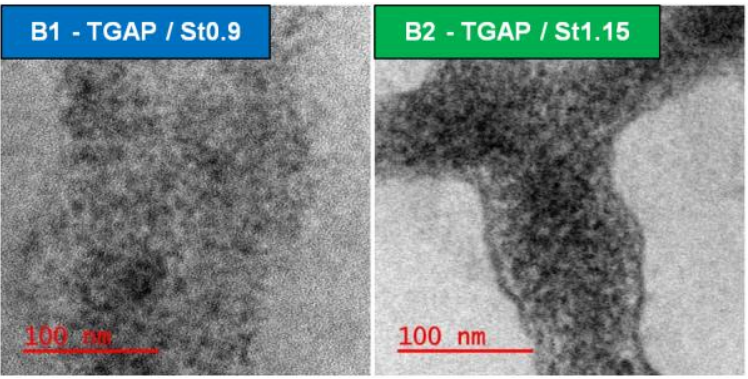

FIGURE 8 TEM images of A1 and A2. Scale bar $100 \mathrm{~nm}$.

However it was not successful for systems A1 and $A 2$. Under a high magnificence some darker zones of a few nanometers are visible on the images but we cannot conclude that it indicates the morphology (Figure 9).

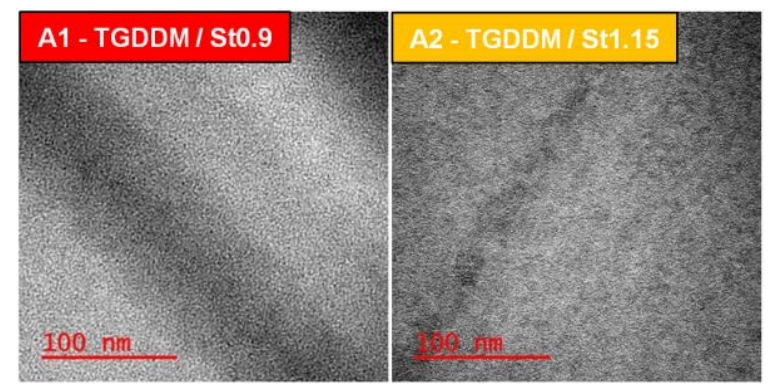

FIGURE 9 TEM images of A1 and A2. Scale bar $100 \mathrm{~nm}$.

We think that the two phases are not pure enough, so that both phases are partially chemically stained by the phosphotungstic acid. It does not permit to bring a sufficient contrast.

Thus, using staining method or dissolution method, no phase separation is observed in systems A. Possibilities are that there is no phase separation so that a homogeneous system is obtained or that morphologies are too small to be observable by SEM. For these systems it was not possible to resolve two different $\mathrm{T}_{\mathrm{g}}$ 's by DSC because the transitions temperatures are very close to each other. For epoxy/TP systems DMTA was used in different studies in the literature in order to detect the temperature of $\alpha$-relaxations during ramp temperature test using a three-point bending method [11] [33] [14] or a single cantilever bending mode [34]. In our study, experiments are run on the different systems during a heating ramp at $2{ }^{\circ} \mathrm{C} \mathrm{min}^{-1}$ with $10 \mathrm{~Hz}$ frequency applied. Tan $\delta$ plot shows evidence of phase separation for the four systems cured with a temperature ramp up to a $180{ }^{\circ} \mathrm{C}$ plateau. Results for systems $\mathrm{A} 1$ and $\mathrm{A} 2$ are represented below (Figure 10). A small shoulder is noticeable on the $\alpha$-relaxation of the TS-rich phase which indicates the $\alpha$-relaxation of the 
TP-rich phase. Indeed, two Gaussian peaks were necessary to fit the relaxations at high temperature with acceptable resolution (error $<7 \%)$ when comparing the area under the experimental curve and the area under the fitted curve.

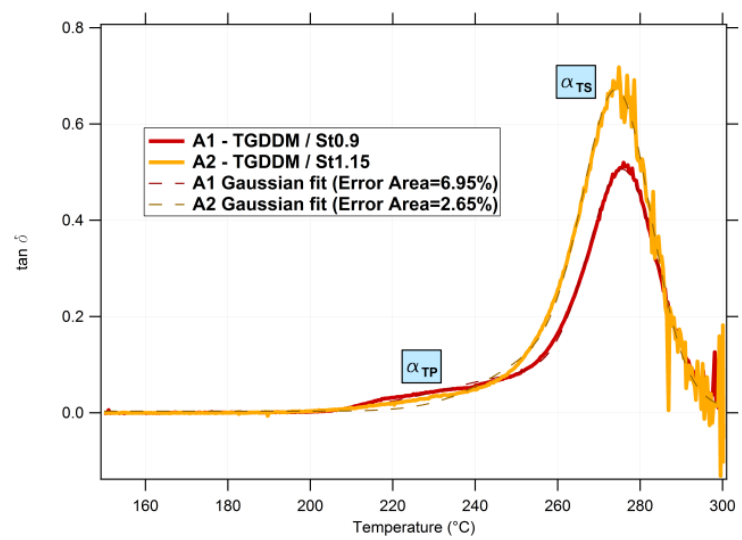

FIGURE 10 DMTA of $A 1$ and $A 2$.

From the fitted curves two relaxation temperatures are given for each curve corresponding respectively to the TP-rich phase and the TS-rich phase. A1 shows $258.3{ }^{\circ} \mathrm{C}$ and $275.9{ }^{\circ} \mathrm{C}$ and A2 $262.0{ }^{\circ} \mathrm{C}$ and $274.5{ }^{\circ} \mathrm{C}$ respectively. The curing temperature is $180^{\circ} \mathrm{C}$.

Size wise, the morphologies for $A 1$ and $A 2$ seem to be in the scale limit of the SEM apparatus. The picture below shows A1 at high magnification after TP-chemical staining (Figure 11).

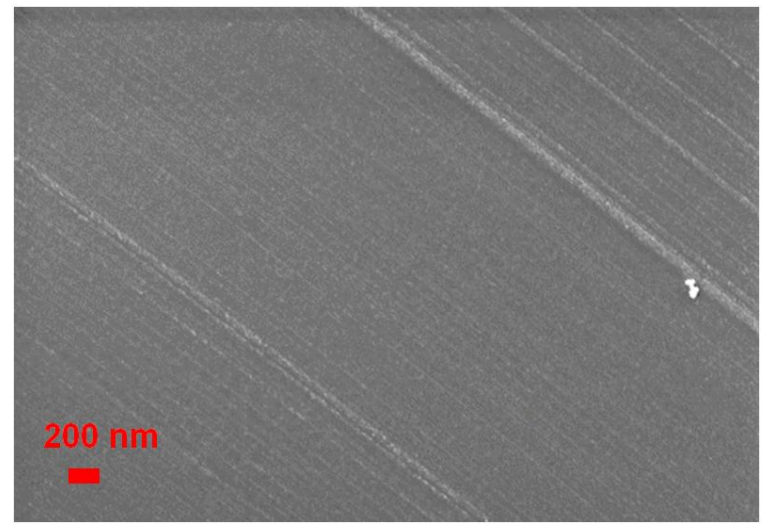

FIGURE 11 SEM observations of system A1 at high magnification. Scale bar $200 \mathrm{~nm}$

Even if we could guess small light nodules (TPrich phase) around $30-50 \mathrm{~nm}$ in a black background (continuous epoxy-rich phase), it is not convincing and the interfaces are not well defined. Thus small-angle neutron scattering has been chosen to investigate nanometer scales. Results for systems $A 1$ and $A 2$ of samples quenched at $20 \%$ of epoxy conversion and of samples after the curing process are presented below (Figure 12).
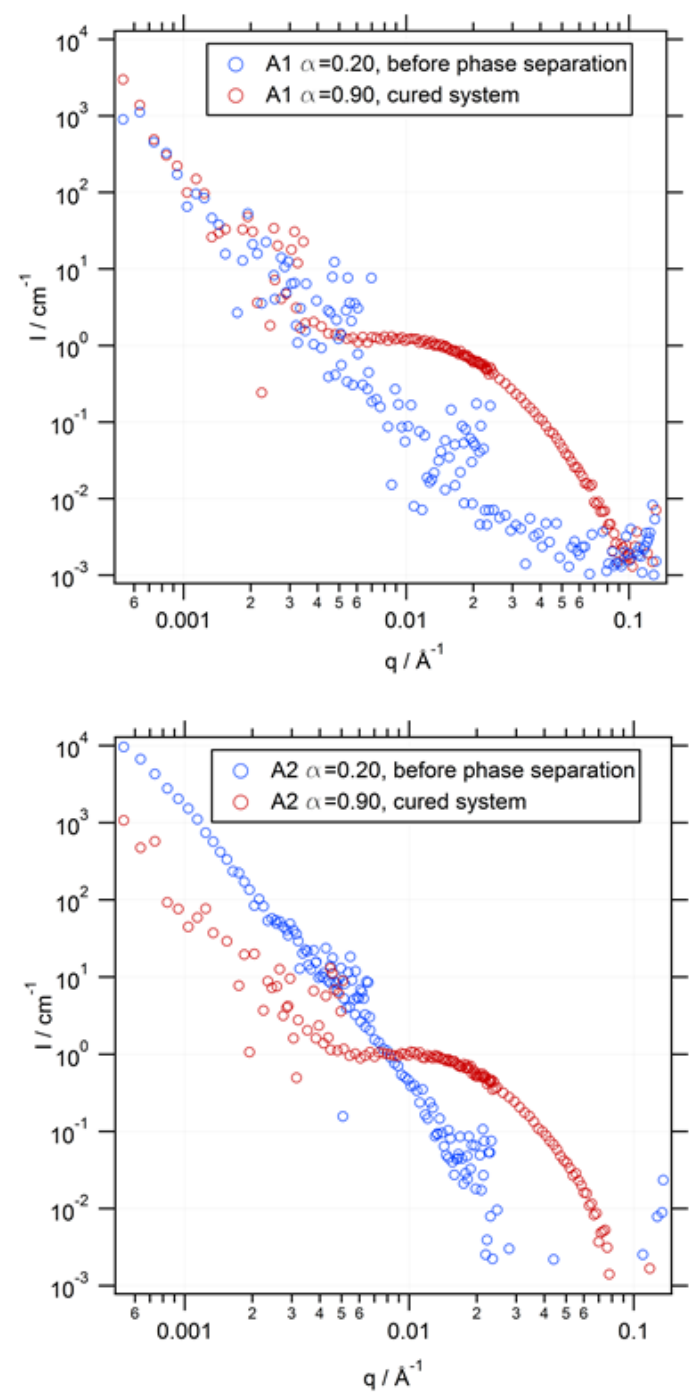

FIGURE 12 SANS intensity of systems $A 1$ and $A 2$, before and after phase separation.

At $20 \%$ of epoxy conversion no morphology can be detected on the scattering data. Note that we estimate the onset of phase separation at around $10 \%$ of epoxy conversion but it is possible that the scattering is not sufficient to identify two phases at this stage of the reaction. 
However the scattered intensity of the final cured systems indicates the presence of two phases corresponding to the well pronounced oscillation at $\mathrm{q}=0.026 \AA^{-1}$. From DMTA and small-angle neutron scattering results we conclude that systems A exhibit phase separation with characteristic size of $24 \mathrm{~nm}$. It is consistent with other studies using TGDDM and polyethersulfone. Fernandez et al. [35] did not see phase separation on TGDDM/DDM/PES system by SEM but DMA showed a small shoulder on the $\alpha$-relaxation peak of the TS which confirmed that phase separation had occurred. Cheng et al. studied TGDDM/TGAP/44'DDS/15 wt\%PES with $\mathrm{OH}$ endgroup through different formulations (along different quantities of each monomer) [31]. Besides no phase separation was seen on SEM, they distinguished nanophases around $50 \mathrm{~nm}$ by Atomic Force Microscopy.

By combining the determination of solubility parameters in initial systems, in situ characterization of the systems along curing and investigation on the final morphologies, a contribution to the understanding of the complex behavior of epoxy/polyethersulfone systems is proposed. Phase separations mechanisms have to be understood according to two characteristics: the onset and the kinetics of phase separation. The onset of phase separation can be predicted by calculating the Flory interaction parameters in the initial system. If phase separation takes place early in the curing process thanks to high $\chi$ values, the morphologies have more time for growing before being arrested by gelation. However, the kinetics of phase separation itself is essential as regards the final morphology. Morphologies in more viscous systems grow more slowly than in less viscous systems. The viscosity is related to the $T_{\mathrm{g}}$ 's of the epoxy and of the thermoplastic and to the temperature at phase separation. In Figure 11 we plot the loss moduli of our different systems during curing (ramp $1^{\circ} \mathrm{C} \mathrm{min}$ $\left.{ }^{1}\right)$. The gel points are indicated by crosses and the onsets of phase separation by squares.

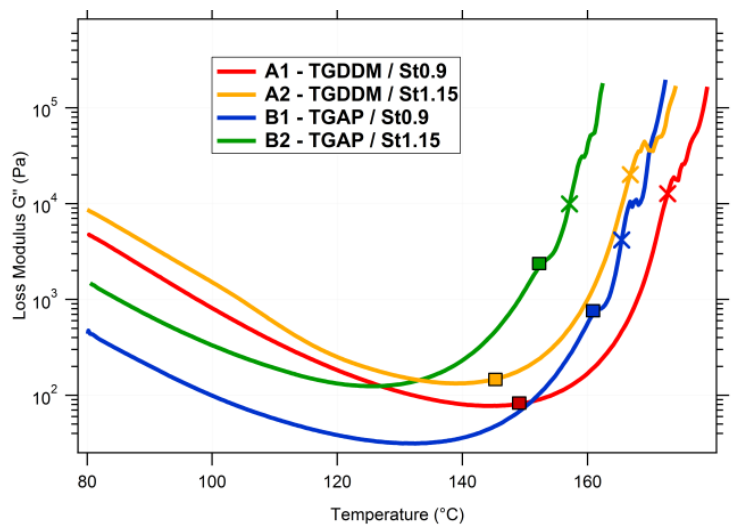

FIGURE 137 Evolution of loss modulus along curing for all systems

Systems B phase separate at a higher epoxy conversion than systems $A$. The elapsed time between phase separation and gelation is about $4 \mathrm{~min}$. We observed above that TP-rich domains in B1 are slightly coarser than in B2. Even if phase separation occurs for both systems at similar epoxy conversion, their kinetics are different. Indeed the viscosity of B1 at phase separation and beyond is almost one decade lower than in B2 which contains a larger quantity of curing agent. As a consequence, we assume that the kinetics of phase separation is faster in B1 than in B2 [1].

Systems A (TGDDM) present a poorer miscibility in initial state than systems $B$. The corresponding value of the $\chi$ parameter between TGDDM and TP is 10 times larger than between TGAP and TP. Phase separation takes place earlier during curing. The epoxy conversion was approximated at $10 \%$ which corresponds to a dimerization of the system. Half of epoxy molecules are attached to an amine. We estimate the time lag between phase separation and gel point to be about 24 min. The kinetics of phase separation is controlled by the high viscosity of the system. Loss moduli for a same epoxy conversion are compared between the different systems, using Figure 2 and 11.

At $20 \%$ of epoxy conversion, the loss moduli for systems $\mathrm{A} 1$ and $\mathrm{A} 2$ are $300 \mathrm{~Pa}$ and $310 \mathrm{~Pa}$ respectively. At the same stage of curing the 
loss moduli for $\mathrm{B} 1$ and $\mathrm{B} 2$ are $63 \mathrm{~Pa}$ and $280 \mathrm{~Pa}$ respectively. At $30 \%$ of epoxy conversion, the loss moduli for systems $\mathrm{A} 1$ and $\mathrm{A} 2$ are $3900 \mathrm{~Pa}$ and $1260 \mathrm{~Pa}$ respectively. At the same stage of curing the loss moduli for B1 and B2 are $180 \mathrm{~Pa}$ and $860 \mathrm{~Pa}$ respectively. We observe thus that the viscosities of A-systems are higher than those of B-systems at the same stage of curing which may be the cause of the smaller sizes of final morphologies in A-systems as compared to B-systems.

\section{CONCLUSIONS}

A methodology for controlling final morphologies in thermoplastic/epoxy blends is applied on four formulations. It starts with the determination of the relative miscibility of the components in initial systems (interaction parameters between epoxy and TP). Then it addresses a kinetic study of epoxy curing by DSC and a thermodynamic study by dynamic rheology with gel point determination and phase separation estimation. The viscosities and the temperatures at phase transition in the different systems are compared. The final morphologies are observed by SEM. A combination of DMTA analysis and neutronscattering study allows determining the size of morphologies of a few tens of nanometers in some samples which are not observable by electron microscopy.

Different morphologies are obtained according to the chosen epoxy monomers and the amount of curing agent, all systems having the same fraction of thermoplastic. Systems composed of
TGDDM present nodules of $40 \mathrm{~nm}$ whereas systems containing TGAP display vermicular morphologies of characteristic width $250 \mathrm{~nm}$ and a few micrometers in length.

The structure of the epoxy monomer used in the formulation influences the onset of phase separation. TGDDM separates earlier during the epoxy curing. At the opposite, TGAP is more miscible with the polyethersulfone and the homogeneous polymer solution is stable until a higher degree of epoxy conversion. The epoxy monomer structure (either tri- or tetrafunctional) controls the gel point and the stopping of the phase separation process. An important parameter is the viscosity of the systems which controls the kinetics of phase separation. TGDDM systems have a higher viscosity than TGAP systems which leads to smaller length scales for the final morphology. Furthermore the stoichiometry also influences the viscosity of systems. The B2 formulation has a higher viscosity than B1 and displays a finer morphology. This study puts forward a combination of methods for investigating both the onset and duration of phase separation and the kinetics of morphology growth of epoxy/polyethersulfone systems.

In conclusion, the morphologies obtained in this study are controlled by the affinity between components and the viscosity of the systems. These parameters will be more systematically studied in future works.

\section{REFERENCES}


[1] J.-P. Pascault, H. Sautereau, J. Verdu, R.J.J. Williams, Thermosetting polymers, Dekker2002.

[2] J. Gotro, R.B. Prime, Thermosets, Encyclopedia of Polymer Science and Technology, John Wiley \& Sons, Inc. 2002.

[3] S. Wu, Control of intrinsic brittleness and toughness of polymers and blends by chemical structure: A review, Polymer International 29(3) (1992) 229-247.

[4] H. Ma, M.A. Aravand, B.G. Falzon, Phase morphology and mechanical properties of polyetherimide modified epoxy resins: A comparative study, Polymer 179 (2019) 10.

[5] M. Jiang, Y. Liu, C. Cheng, J. Zhou, B. Liu, M. Yu, H. Zhang, Enhanced mechanical and thermal properties of monocomponent high performance epoxy resin by blending with hydroxyl terminated polyethersulfone, Polymer Testing 69 (2018) 302-309.

[6] I. Martinez, L. Martin, A. Eceiza, P.A. Oyanguren, I. Mondragon, Phase separation in polysulfonemodified epoxy mixtures. Relationships between curing conditions, morphology and ultimate behavior, Polymer 41 (2000) 1027-1035.

[7] C.B. Bucknall, I.K. Partridge, Addition of polyethersulphone to epoxy resins, British Polymer Journal 15(1) (1983) 71-75.

[8] J.-P. Pascault, Rubber- and thermoplastic-modified polymer networks. Phase separation process induced by polymerization and polycondensation, Macromolecular Symposia 93(1) (1995) 43-51. [9] P.A. Oyanguren, M.J. Galante, K. Andromaque, P.M. Frontini, R.J.J. Williams, Development of bicontinuous morphologies in polysulfone-epoxy blends, Polymer 40(19) (1999) 5249-5255.

[10] M. Rico, J. López, B. Montero, R. Bellas, Phase separation and morphology development in a thermoplastic-modified toughened epoxy, European Polymer Journal 48(10) (2012) 1660-1673.

[11] G.T. Emmerson, Phase separation and mechanical properties of epoxy/thermoplastic blends, Durham University, Durham University, 2003.

[12] M. Rico, J. López, B. Montero, R. Bouza, F.J. Díez, Influence of the molecular weight of a modifier on the phase separation in an epoxy thermoset modified with a thermoplastic, European Polymer Journal 58 (2014) 125-134.

[13] Y. Rosetti, P. Alcouffe, J.-P. Pascault, J.-F. Gérard, F. Lortie, Polyether Sulfone-Based Epoxy Toughening: From Micro- to Nano-Phase Separation via PES End-Chain Modification and Process Engineering, Materials (Basel, Switzerland) 11(10) (2018) 1960.

[14] I. Blanco, G. Cicala, O. Motta, A. Recca, Influence of a selected hardener on the phase separation in epoxy/thermoplastic polymer blends, Journal of Applied Polymer Science 94(1) (2004) 361-371.

[15] W. Ballout, P. Van Velthem, D. Magnin, E. Henry, M. Sclavons, T. Pardoen, C. Bailly, Specific influence of polyethersulfone functionalization on the delamination toughness of modified carbon fiber reinforced polymer processed by resin transfer molding, Polymer Engineering \& Science 59(5) (2019) 996-1009.

[16] M. Kurata, H. Fujita, Thermodynamics of Polymer Solutions, Harwood Academic Publishers1982.

[17] R.J.J. Williams, B.A. Rozenberg, J.-P. Pascault, Reaction-induced phase separation in modified thermosetting polymers, Polymer Analysis Polymer Physics, Springer Berlin Heidelberg, Berlin, Heidelberg, 1997, pp. 95-156.

[18] B.S. Kim, T. Chiba, T. Inoue, Phase separation and apparent phase dissolution during cure process of thermoset/thermoplastic blend, Polymer 36(1) (1995) 67-71.

[19] B.S. Kim, T. Chiba, T. Inoue, A new time-temperature-transformation cure diagram for thermoset/thermoplastic blend: tetrafunctional epoxy/poly(ether sulfone), Polymer 34(13) (1993) 28092815.

[20] P.J. Flory, Principles of Polymer Chemistry, Cornell University Press1953.

[21] K.L. Hoy, C. Union Carbide, Solvents, D. Coatings Materials, Research, D. Development, The Hoy tables of solubility parameters, Union Carbide Corp., Solvents \& Coatings Materials, Research \& Development Dept., South Charleston, W. Va., 1985. 
[22] P.G. de Gennes, C.U. Press, Scaling Concepts in Polymer Physics, Cornell University Press1979.

[23] M. Rubinstein, R.H. Colby, Polymer physics, Oxford university press New York2003.

[24] J.O. Swanson, E.W. Fowler, S.M. Wand, M. Pramanik, J.W. Rawlins, Investigation of network development and properties in multifunctional epoxy resins using 3,3'-diaminodiphenylsulfone., School of Polymers and High Performance Materials, 2010.

[25] R. Sellak, Elaboration et caractérisation d'une résine thermodurcissable conductrice, Université du Maine, 2013.

[26] Q. Voleppe, T. Pardoen, C. Bailly, Interdiffusion and phase separation upon curing in thermosetthermoplastic interphases unravelled by the characterization of partially cured systems., Polymer 106 (2016) 120-127.

[27] H.H. Winter, F. Chambon, Analysis of Linear Viscoelasticity of a Cross-Linking Polymer at the Gel Point, Journal of Rheology 30 (1986) 367-382.

[28] H.H. Winter, Can the gel point of a cross-linking polymer be detected by the $G^{\prime} \quad-G^{\prime \prime}$ crossover?, Polymer Engineering \& Science 27(22) (1987) 1698-1702.

[29] G. Cicala, I. Blanco, A. Latteri, L. Oliveri, A. Recca, Influence of 1,5-naphthalenediamine on thermomechanical properties of epoxy/copolyethersulphone blends, Polymer Engineering \& Science 53(1) (2013) 219-225.

[30] G. Cicala, A. Mamo, G. Recca, C.L. Restuccia, Study on epoxy/thermoplastic blends based on the addition of a novel aromatic block copolymer, Polymer Engineering \& Science 47(12) (2007) 2027-2033. [31] X. Cheng, Q. Wu, S.E. Morgan, J.S. Wiggins, Morphologies and mechanical properties of polyethersulfone modified epoxy blends through multifunctional epoxy composition, Journal of Applied Polymer Science 134(18) (2017).

[32] E. Girard-Reydet, H. Sautereau, J.P. Pascault, P. Keates, P. Navard, G. Thollet, G. Vigier, Reactioninduced phase separation mechanisms in modified thermosets, Polymer 39(11) (1998) 2269-2279. [33] I. Martinez, M.D. Martin, A. Eceiza, P. Oyanguren, I. Mondragno, Phase separation in polysulfonemodified epoxy mixtures. Relationships between curing conditions, morphology and ultimate behavior., Polymer (2000).

[34] G. Cicala, S. Mannino, A. Latteri, G. Ognibene, G. Saccullo, Effects of mixing di- and tri-functional epoxy monomers on epoxy/thermoplastic blends, Advances in Polymer Technology 37(6) (2018) 18681877.

[35] B. Fernández, A. Arbelaiz, E. Diaz, I. Mondragon, Influence of polyethersulfone modification of a tetrafunctional epoxy matrix on the fracture behavior of composite laminates based on woven carbon fibers, Polymer Composites 25(5) (2004) 480-488. 


\section{GRAPHICAL ABSTRACT}

Eléonore Mathis, Marie-Laure Michon, Claude Billaud, Pauline Grau, Anthony Bocahut, Caroll Vergelati, Didier R. Long ${ }^{1}$

\section{THERMOSET MODIFIED WITH POLYETHERSULFONE: CHARACTERIZATION AND CONTROL OF THE MORPHOLOGY.}

The control of morphology is challenging to the control of mechanical properties in polyethersulfonemodified epoxy resin for composites application. A combination of methods for characterizing the kinetic and thermodynamic behavior as well as the final morphology is applied on four systems. Significant differences in final morphology size and type is assigned to the influence of the interaction parameter and the viscosity.

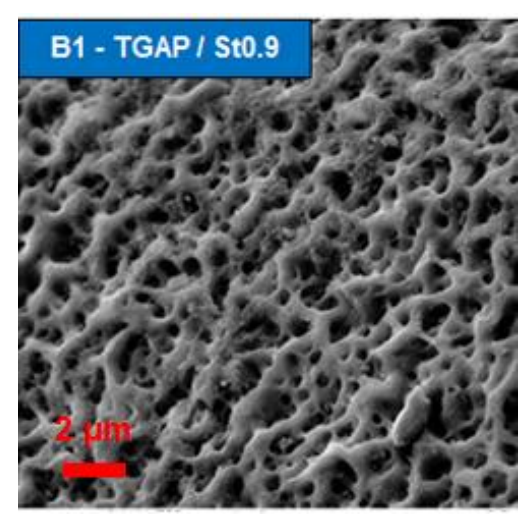

